\title{
Perceptions and Knowledge About the MenB Vaccine Among Parents of High School Students
}

\author{
Eric Richardson ${ }^{1} \cdot$ Kathleen A. Ryan ${ }^{3} \cdot$ Robert M. Lawrence $^{3} \cdot$ Christopher A. Harle $^{1}$. Alyson Young ${ }^{1}$. \\ Melvin D. Livingston ${ }^{4} \cdot$ Amit Rawal $^{5} \cdot$ Stephanie A. S. Staras ${ }^{1,2}$
}

Accepted: 27 November 2020 / Published online: 2 January 2021

(c) The Author(s) 2021

\begin{abstract}
Serogroup B meningococcal disease (MenB) causes almost $60 \%$ of meningitis cases among adolescents and young adults. Yet, MenB vaccine coverage among adolescents remains below $10 \%$. Since parents are the primary medical decision makers for adolescents, we examined MenB vaccination rates and parent attitudes about meningitis and the MenB vaccine. In 2018, in conjunction with a county-wide, school-based immunization campaign, we conducted a mixed methods study among parents of 16- to 17-year-olds. We facilitated focus groups asking parents about their knowledge of meningitis and reactions to educational materials and sent behavioral surveys based on Health Belief Model constructs to parents through the county high school system. Parents in three focus groups $(n=8$; participation rate $=13 \%)$ expressed confusion about their child's need to receive the MenB vaccine in addition to the meningococcal conjugate vaccine (MenACWY), but conveyed strong trust in their physicians' recommendation. Among survey participants $(n=170), 70(41 \%)$ had heard of the MenB vaccine. Among those 70 parents, the most common barriers to vaccination were concerns about side effects (55\%) and uncertainty of susceptibility due to receipt of the MenACWY vaccine (30\%). The percentage of teens that received at least one dose of the MenB vaccine was $50 \%(n=35)$ by parent report and $23 \%(n=16)$ by state vaccination records. Parents demonstrated uncertainty and confusion about the MenB vaccine particularly due to the existence of another meningitis vaccine and limited health care provider recommendations. Confirmatory studies of parent confusion about the MenB vaccine are needed to develop interventions.
\end{abstract}

Keywords Immunization $\cdot$ MenB $\cdot$ Community intervention $\cdot$ Cross-sectional survey $\cdot$ Focus group

Between 2014 and 2016, serogroup B (MenB) was the cause of $58 \%$ of the 166 cases of meningococcal disease in persons aged 18 to 24 years in the United States [1,2]. Notably, serogroup B is the primary cause of organization-based outbreaks and has an approximate case fatality rate of $7 \%[3$,

Stephanie A. S. Staras

sstaras@ufl.edu

1 Department of Health Outcomes and Biomedical Informatics, College of Medicine, University of Florida, Gainesville, FL, USA

2 The Institute for Child Health Policy, University of Florida, 2004 Mowry Road, Room 2238, Gainesville, FL 32610, USA

3 Department of Pediatrics, College of Medicine, University of Florida, Gainesville, FL, USA

4 Rollins School of Public Health, Emory University, Atlanta, GA, USA

5 Avnee Foundation, Gainesville, FL, USA
4]. The best strategy to prevent MenB disease is vaccination with one of the two vaccines licensed for use among 10- to 25-year-olds [5]. As of 2016, the Advisory Committee on Immunization Practices' (ACIP) recommends either of the MenB vaccines as a shared clinical decision (Category B) for healthy individuals between 16- and 23-years (preferred at 16- to 18-years) and routine (Category A) to individuals ages $\geq 10$-years-old who are at increased risk (e.g., persistent complement component deficiencies, complement inhibitor use, asplenia or during disease outbreaks) [6]. In 2019, MenB vaccine initiation (i.e., received at least one dose) among 16- to 18-year-olds in the United States was an estimated $21 \%$ [7].

Since parents are often the primary decision makers for adolescent vaccines, parental acceptance of the MenB vaccine is likely key to increasing uptake [8]. A handful of studies performed outside the United States have assessed parents' perceptions about the MenB vaccine and found most 
parents have heard of meningitis (80-86\%) and wanted their child to receive the MenB vaccine (62-64\%) [9-12]. Within the United States, to our knowledge, only two studies examined parents' awareness about and intention for their highschool-aged children to receive the MenB vaccine [13, 14]. Among a 2017 convenience sample of 445 parents of Minnesota high school students, $72 \%$ had heard of meningitis, but only $20 \%$ were aware of the MenB vaccine [13]. In this study, compared to parents who had not heard of the vaccine, the odds of parents' intent to have their child vaccinated increased nearly fourfold among parents aware of the MenB vaccine and threefold among parents at least somewhat concerned about meningitis. A second study included a random sample of 619 parents across the US, and weighted results to correspond with the US population [14]. Based on the weighted results, an estimated $43 \%$ of US parents are aware of the MenB vaccine, and among those only $45 \%$ intend to get the MenB vaccine. As such, only an estimated 19\% of parents intended to receive the MenB vaccine.

Theory and vaccination studies suggest, however, that parental awareness of a vaccine is not sufficient to lead to vaccination [15-19]. A widely recognized behavioral theory, the Health Belief Model suggests parents' decision to get their child vaccinated is influenced by: (1) perceived threat and severity of the disease, (2) belief the vaccine will offer benefits (e.g., preventing meningitis), (3) perceived barriers to getting the vaccine, (4) self-efficacy to complete the behavior, and (5) a cue to action (e.g., a recommendation from a healthcare provider). Guided by the Health Belief Model, we aimed to identify rates and parent attitudes about MenB vaccination for 16- to 17-year old adolescents. We chose 16- to 17-year-olds because they are within the recommended age of vaccination and are dependent upon parent consent to receive the vaccine. Understanding more about parent attitudes could help develop and adapt interventions to increase MenB vaccination and ultimately reduce MenB meningitis cases.

\section{Methods}

\section{Research Design}

We used a mixed method design with parent focus groups and a cross-sectional survey to understand parents' attitudes about the MenB vaccine. The study included parents of 16- to 17-year-olds in a north-central Florida county, Alachua. The University of Florida Institutional Review Board approved study procedures.

\section{Focus Groups}

In February 2018, we invited parents of 16- to 17-year-old adolescents residing in Alachua County who had visited a University of Florida primary care clinic in the past year to participate in focus groups. We called parents up to three times at phone numbers obtained through a registry of University of Florida patients who consented to receiving research invitations [20]. We reminded agreeing parents on the day before the focus groups.

Immediately before the focus groups, we orally explained the informed consent to the group of parents. Parents who agreed to participate signed the informed consent form and participated in the focus groups. A trained moderator conducted the groups in a private room by following a semi-structured guide. Questions included parents' opinions about meningococcal disease, the MenB vaccine, and the content and design of publicly available education materials from the Centers for Disease Control and Prevention, Immunization Action Coalition, and National Meningitis Association [21-23]. Incentives included a meal and \$25. Focus groups were audiorecorded and transcribed.

\section{Qualitative Analysis}

We conducted a thematic analysis of focus group transcripts using Nvivo qualitative data analysis software Version 12 [24]. Between December 2019 and March 2020, two members of the research team (AY, ER) analyzed the focus group transcripts using thematic analysis from three rounds of independent coding [25]. In the first coding round, we created two primary themes. In the second coding round, we assigned each response to a primary theme and identified secondary themes. After conferring about the saliency of the secondary themes, we created a coding framework. In the third coding round, we applied the coding framework to the transcripts. We selected specific quotes to represent each coded theme.

\section{Cross-Sectional Survey}

In March, May and November 2018, we invited parents to complete a survey through the county school system. Surveys were collected before and after a county-wide, school-based vaccination campaign. In April and May 2018, the Florida Department of Health sent vaccination consent forms to parents of students in 11th and 12th grades (approximate ages 16 to 18 years) via the county school superintendent's office distribution list. With a signed parent consent, students received immunizations for 
MenB, meningococcal conjugate vaccine (MenACWY), human papillomavirus (HPV), and hepatitis A on specified days at county high schools.

In March 2018, prior to the vaccination campaign, we randomly selected one-third of the county's 10-12th grade English classes to distribute 1250 survey packets including a waiver of documentation of consent letter, paper survey, $\$ 1$ cash, and a stamped return envelope. English teachers distributed packets to students in assigned classes. The waiver of documentation of consent letter explained the purpose of the study and all elements of informed consent. The letter instructed parents that returning the completed survey would be considered as providing consent. Returned paper-based surveys were double entered into Research Electronic Data Capture (REDCap) [26, 27].

In May 2018, due to low participation rates in March 2018, we sent a second invite to parents. Parents of high school students were invited via the county school superintendent's office distribution list to complete the survey using either their packet or a link to an online, REDCap survey. Parents who completed the survey online signed an electronic consent form in REDCap.

In November 2018, while originally planned as a time for a follow-up survey of spring participants, due to low participation rates in March and May, we again invited all high school parents to complete the survey. The superintendent's office used their distribution list to invite all parents to complete the online, REDCap survey.

\section{Survey Constructs}

Using items adapted from previous vaccine surveys (e.g., the Health Information National Trends Survey and the National Survey of Children's Health), we assessed the theoretical constructs of the Health Belief Model [19, 28-30]. We measured perceived severity by participants' familiarity with meningitis and if it posed a serious threat to their child. We asked participants if meningitis caused four outcomes (loss of limbs, death, brain damage, and cancer). We created a composite knowledge score representing the total number of correctly identified outcomes ranging from 0 (all incorrect) to 4 (all correct).

Among parents who had heard of the MenB vaccine, we measured the perceived benefits and barriers of the MenB vaccine. A potential barrier considered was prior receipt of the MenACWY vaccine because of its potential confusion with MenB and its Category A recommendation for routine vaccination at 11 years and a second dose at 16 years from the ACIP [31]. Lastly, we assessed provider recommendations using three questions from prior surveys [19, 32]: whether a healthcare provider discussed, recommended, and, if recommended, expressed importance.

\section{MenB Vaccine Initiation}

We assessed vaccine initiation with two imperfect data sources: parent-report and vaccination records in the Florida immunization registry [33]. Adapted from the National Survey of Children's Health [28], we asked parents to report whether their adolescent received MenACWY vaccine, meningococcal B (MenB) vaccine, both, or not sure. To objectively measure vaccine initiation, we requested parents provide identifying information for the child. Using this information, the Florida Department of Health staff obtained records of MenB vaccine doses received from the state immunization registry by the approximate date parents completed the survey.

\section{Quantitative Analysis}

We conducted a descriptive analysis of the survey data. To maintain independence of responses we used the first response for parents who completed the survey more than once $(n=5)$. We compared the sensitivity and specificity of parent-reported vaccines to the Florida immunization registry records using a crosstab analysis. Analyses were conducted using SAS software version 9.4 (SAS Institute, Inc., Cary, NC).

Because of the small number of respondents prior to the Department of Health campaign $(n=40)$, we did not attempt to evaluate changes in parent attitudes or vaccination rates based on the campaign. We were, however, concerned that parents who completed the survey after the vaccination campaign may have been influenced by the campaign. To assess for this potential influence, we compared knowledge of the MenB vaccine in parents who responded prior to the campaign (March 2018) to those who responded after the campaign (May and November 2018).

\section{Results}

\section{Focus Groups}

Among the 61 parents with contact information in the research registry, 8 attended one of three focus group sessions (the groups included 3, 3, and 2 participants, respectively) for a $13 \%$ participation rate. Participating parents were mainly educated with at least a college degree (5/8), non-Hispanic white (5/8), and between 36- and 52-yearsold (5/8). All of the parents reported that their child had an established primary care pediatrician. Focus groups lasted an average of $70 \mathrm{~min}$ (Range 63-84 $\mathrm{min}$ ).

Most parents were aware of meningitis or meningococcal disease. Common descriptions about the disease included 'serious,' 'fast,' and 'devastating.' For example, one parent 
described meningitis as: "I mean when you see - hear the stories about kids in college, amputations, all these consequences and that, right, then you realize, well, it may be a small chance that it's going to happen, but when it happens it's just devastating."

When reviewing MenB vaccine educational materials, parents expressed confusion about meningococcal vaccines. Parents asked why children needed to get a second vaccine for meningitis and why serogroup B had a separate vaccine. Exemplar quotations were:

Because they provide routine vaccinations at age 11 to 12 for all the rest, right? So it's like maybe, well, my child's good enough, they've got that vaccine, so why do I have to give him another vaccine for another type of meningitis?

It's just one serial group that it covers. And it says other meningococcal vaccines are recommended to help protect against the ACWY. So I'm just wondering why is this one - is this one just more common?

By and large, parents reported they would follow the advice of their pediatrician regarding vaccines. For example, one parent said: "I mean, not being in the medical field, I feel like that's something I'm going to trust my pediatrician to choose and talk to me about the right medicine to use."

\section{Survey Respondents}

Overall, 170 caregivers of 16-17-year-olds completed our survey at three time points: 40 in March (prior to the Department of Health campaign), 46 in May, and 84 in November 2018 Most respondents (97\%) were parents; thus, for the remainder of the paper, we refer to caregivers as parents. Parents were an average of 49 years-old (range 34-69 years), and majority were non-Hispanic white (78\%), educated (28\% receiving a Bachelor's degree and 33\% receiving a Master's degree or above), married (75\%), and had insurance for their child (96\%) (Table 1). In our sample, approximately half $(56 \%)$ had a female 16 - to 17 -year-old.

\section{Awareness of Meningitis and the Meningitis Vaccines}

Among the 170 parents, almost all (94\%) had heard of meningococcal disease or meningitis. When asked about meningococcal vaccines, parents were more likely to report having heard about MenACWY (61\%) than the MenB vaccine $(40 \%)\left(\chi^{2}=79.19 \mathrm{p}<0.001\right)$. We found no evidence of temporal increases in awareness potentially
Table 1 Demographic characteristics of parents who completed the survey

\begin{tabular}{lc}
\hline & Participants (N=170) \\
& $\mathrm{N}(\%)$ \\
\hline Race/ethnicity & \\
White non-Hispanic & $128(78 \%)$ \\
Hispanic or Latino/a & $13(8 \%)$ \\
African American or Black (non-Hispanic) & $10(7 \%)$ \\
Other & $9(6 \%)$ \\
Education & \\
High school diploma or less & $20(5 \%)$ \\
Vocational degree & $5(9 \%)$ \\
Associates degree & $34(17 \%)$ \\
Bachelor's degree & $39(28 \%)$ \\
Master's degree or higher & $57(33 \%)$ \\
Child's health insurance & \\
Through current or former employer & $89(71 \%)$ \\
Medicaid, medical assistance & $12(10 \%)$ \\
Other & $8(5 \%)$ \\
Purchased directly from insurance company & $7(6 \%)$ \\
Not covered by any insurance & $5(4 \%)$ \\
Marital status & \\
Married & $117(75 \%)$ \\
Divorced & $16(11 \%)$ \\
Never married & $8(5 \%)$ \\
Widowed & $5(3 \%)$ \\
Not married, living with partner & $5(3 \%)$ \\
Separated & $4(3 \%)$ \\
\hline &
\end{tabular}

due to the Department of Health campaign that would preclude combining parent responses: similar percentages of parents had heard of the MenB vaccine in March 2018 $(35 \%)$ as in May and November $2018(42 \%)\left(\chi^{2}=2.13\right.$ $\mathrm{p}=0.71)$.

\section{Perceived Seriousness of Meningitis}

Regardless of having heard about the MenB vaccine, almost all (96\%) parents agreed that meningitis posed a serious threat to their child, and correctly identified death and brain damage as meningitis outcomes (Table 2). About half of parents correctly identified that cancer was not an outcome of meningitis. Correct identification of loss of limbs as an outcome of meningitis was more common among parents that heard of MenB vaccine (41\%) than parents who had not heard of the MenB vaccine (21\%) $(p=0.01)$. When comparing overall composite knowledge scores, the parents who had heard of the MenB vaccine had a 0.3 higher average score than parents that had not heard of the MenB vaccine $(\mathrm{p}=0.03)$. 
Table 2 Differences in knowledge about meningitis based on awareness of MenB vaccine

\begin{tabular}{lllc}
\hline $\begin{array}{l}\text { Do you think meningococcus } \\
\text { can cause... }\end{array}$ & $\begin{array}{l}\text { Heard of MenB vaccine } \\
(\mathrm{N}=66) \\
\text { Answered correctly }\end{array}$ & $\begin{array}{l}\text { Not heard of MenB vaccine } \\
(\mathrm{N}=80) \\
\text { Answered correctly }\end{array}$ & 0.88 \\
\hline Death & $65(98 \%)$ & $74(93 \%)$ & 0.85 \\
Brain or nerve problems & $63(95 \%)$ & $77(96 \%)$ & 0.40 \\
Cancer & $40(61 \%)$ & $43(54 \%)$ & 0.01 \\
Loss of limbs & $27(41 \%)$ & $17(21 \%)$ & 0.03 \\
Mean Composite Score & 3.0 & 2.7 &
\end{tabular}

\section{Perceived Benefits, Barriers, and Susceptibility}

Among parents who had heard of the MenB vaccine $(n=70)$, most parents agreed that the benefits of the MenB vaccine included safety and effectiveness (Fig. 1). Perceived barriers centered on side effects: $19 \%$ were concerned and $36 \%$ were unsure. Since their child had received the MenACWY vaccine, about one-quarter of parents $(28 \%)$ were unsure, and $2 \%$ thought their child did not need the MenB vaccine.

\section{Provider Recommendation}

Most parents (86\%) reported their child attended at least one preventative visit in the previous year. Among parents who heard of the MenB vaccine $(n=70), 81 \%$ indicated that a healthcare provider spoke to them about the MenB vaccine, and $75 \%$ reported that their doctor recommended it. A majority $(71 \%)$ indicated that receiving the vaccine was important to their provider. Assuming that all parents who have not heard of MenB did not receive a recommendation from their healthcare provider (0/100), only $31 \%$ (52/170) of participating parents may have received a recommendation for the MenB vaccine by a healthcare provider.

\section{MenB Vaccine Initiation}

All parents that had heard of the MenB vaccine $(n=70)$ provided their child's information to allow us to obtain records from the state vaccine registry. The Department of Health staff found vaccination records for all 70 children. Based on the state immunization registry records, 16/70 (23\%) of the 16- to 17-year-olds had received at least one dose of the MenB vaccine. But, based on parent-report, 35/70 (50\%) had received at least one dose of the MenB vaccine. To estimate the MenB vaccination rates for all respondents, we assumed that none of the children of parents who were unaware of MenB vaccine had received the vaccine. Under this assumption, between $9 \%(16 / 170)$ and $22 \%$ (37/170) of the 16- to 17-year-olds had received at least one dose of the MenB vaccine. Among the 16 adolescents with records of at least one dose of the MenB vaccine, $56 \%$ completed the series.

When we compared parent-reported vaccination to the immunization registry, the child's MenB vaccine status agreed just over half of the time (Table 3). Among the 16 children who had records of receiving the MenB vaccine, only ten parents reported the child had received the vaccine (sensitivity $=0.63$ ). Among the 54 children without records for the MenB vaccine, 25 parents reported their child had received the vaccine (specificity $=0.46$ ).
Fig. 1 Parents' attitudes about the MenB vaccine $(n=70)$

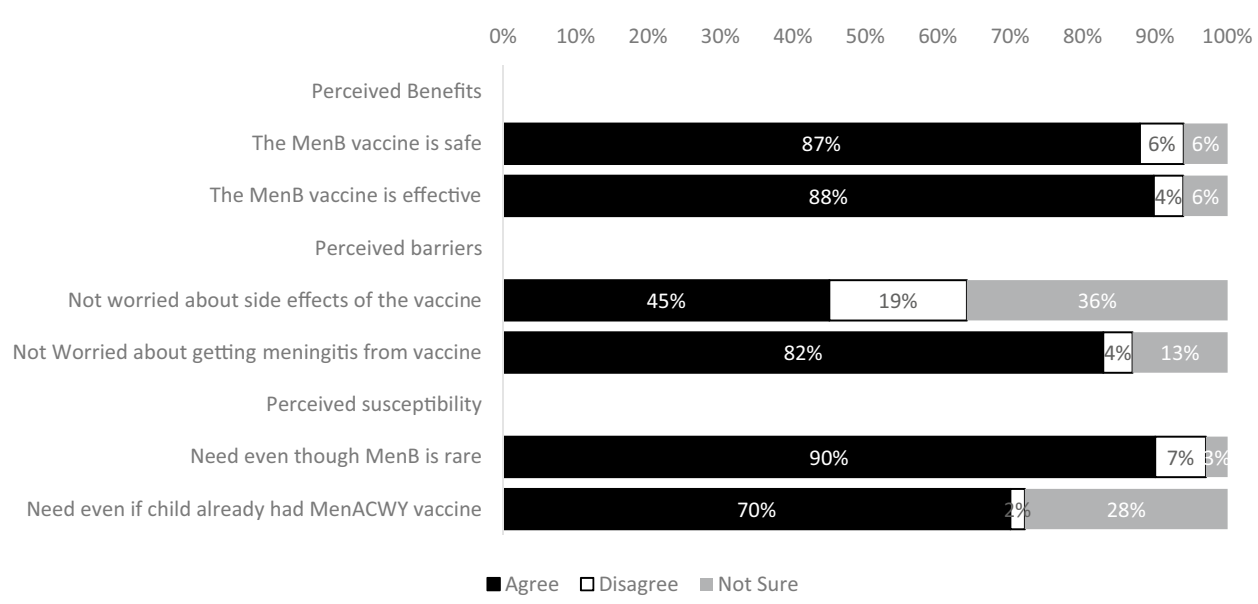


Table 3 Sensitivity and specificity of parents'-report MenB vaccine initiation $(\mathrm{N}=70)$

\begin{tabular}{|c|c|c|c|c|}
\hline & \multicolumn{4}{|c|}{ State immunization registry } \\
\hline & Vaccine & No vaccine & Total & \\
\hline \multicolumn{5}{|c|}{ Parents'-report } \\
\hline Vaccine & 10 & 25 & 35 & $\mathrm{PPV}=0.29$ \\
\hline No vaccine & 6 & 29 & 35 & $\mathrm{NPV}=0.17$ \\
\hline \multirow[t]{2}{*}{ Total } & 16 & 54 & 70 & \\
\hline & Sensitivity $=0.63$ & Specificity $=0.46$ & & $\begin{array}{l}\text { Correct } \\
\text { classifica- } \\
\text { tion }=56 \%\end{array}$ \\
\hline
\end{tabular}

\section{Discussion}

In addition to typical barriers for vaccination, parents expressed uncertainty about the relationship between the two meningitis vaccines; a sentiment that potentially impedes MenB vaccine uptake. Similar to other new vaccines, only half of the parents had heard of the MenB vaccine and the majority of those who heard about it were concerned about side effects [34]. Unlike other vaccines, parents expressed concerns about susceptibility to meningitis and the need for the MenB vaccine since their child already received a meningitis vaccine, MenACWY. Finally, while parents expressed confusion about the need for an additional meningitis vaccine, they ultimately expressed trust in their physician.

As with other adolescent vaccines when newly recommended, a majority of parents were not aware of the MenB vaccine. For example, in 2007, only $42 \%$ of parents could identify the MenACWY vaccine as a recommended vaccine [34]. By 2017, the awareness of the MenACWY vaccine increased to $75 \%$ [13]. Some researchers attributed low parental awareness of MenACWY to infrequent physician recommendations and missed clinical opportunities [35]. A similar phenomena may be occurring for MenB vaccine. Nearly all parents in our study reported that their child had at least one preventive visit during the past year, and therefore, had an opportunity to receive the MenB vaccine. Under the assumption that none of the parents who were unaware of the MenB vaccine had received a provider recommendation or their child had received the vaccine, an estimated $31 \%$ of parents received a physician recommendation and 9-22\% had initiated the MenB vaccine. These results suggest that $70-80 \%$ of 16 - to 17 -year-olds had missed opportunities to receive the MenB vaccine.

The large proportion of parents concerned about MenB vaccine side effects is also similar to other adolescent vaccines suggesting that between 41 and $73 \%$ of parents are concerned about vaccine side effects $[19,36]$. Literature on vaccine hesitancy shows that concern about side effects is a significant barrier to parents deciding whether their adolescent receives a vaccine [37-39]. As such, the concern about
MenB vaccine side effects may reflect a general concern about vaccines.

Parents who had heard of the MenB vaccine were unsure about their child's susceptibility to MenB: an important predictor of vaccination according to the Health Belief Model [18]. Over two times as many parents were concerned about their child's susceptibility to MenB compared to MenACWY ( $<10 \%$ of a nationally representative sample of parents) $[40,41]$. The higher percentage concerned and reports from our focus groups suggest that the time sequencing of the meningococcal vaccines (MenACWY recommended at 11to 12-year-olds and MenB recommended for 16- to 17-yearolds) may inadvertently cause parents to perceive MenB vaccine as the other or less important meningitis vaccine.

Parents' confusion about the relationship between the two vaccines is also reflected in the low sensitivity and specificity we found between parent-reported and immunization registry recorded MenB vaccination. The 56\% match found between parent report and immunization records is well below other studies showing $78 \%$ to $88 \%$ match rates between parent report and immunization records for adolescent vaccines [40-42]. Parents may think receiving the meningococcal conjugate vaccine will protect their child from meningitis in general or simply be unaware that there are two distinct meningococcal vaccines.

The observed parents' confusion about the MenB vaccine may also be a reflection of physician uncertainty. A national survey found that over half (59\%) of pediatricians found it difficult to explain category B recommendations to parents, and as many as $12 \%$ provided information about the conjugate vaccine when recommending the MenB vaccine [43, 44]. The resulting unclear communication limits the inherent power of physician vaccine recommendations [45-47].

Our study includes three important limitations. First, our survey sample size was small due low participation rates. The sample size was reduced further for many questions since only half of parents had heard of the MenB vaccine. Moreover, our sample may not be representative of parents of Alachua county high school students or other areas of the United States. Second, because we had low response rates to our initial survey collection, we did not 
analyze separate time points including pre-/post- differences of the Florida Department of Health vaccination campaign. Without evidence of a temporal increase in awareness of the MenB vaccine during our study period, we analyzed the data as a cross-sectional evaluation of parents' attitudes and adolescents' vaccination rates. Third, our focus groups included a limited number of parents and we primarily targeted understanding the acceptability of nationally available education materials. However, these materials did include information relevant to perceptions about the MenB vaccine.

This study has three important strengths. First, our study is one of a few for MenB vaccine that specifically targets parents of high-school-aged children [13]. This understudied group of late adolescents represents an important opportunity for teens to receive the MenB vaccine before college entry and increased exposure. Second, the study includes an objective measure of MenB vaccine receipt. Previous research on parents' attitudes about the MenB vaccine relied on self-report measures about parental intent to vaccinate their child [13]. Third, we included both quantitative and qualitative data to examine parents' perceptions and knowledge. The qualitative data provided nuanced information about parents' confusion about the vaccine and trust in the provider, while the survey data provided a sense of the relative frequency of specific hesitations.

Among parents of 16- to 17-year-olds, uncertainty about the relationship between the two meningitis vaccines likely presents a barrier to MenB vaccine uptake. While several of the parent concerns were similar to those expressed for other vaccine, unlike other adolescent vaccines, parents were unsure about the susceptibility of their child to MenB primarily because of the MenACWY vaccine. Future studies should consider how physicians can discuss the MenACWY and MenB vaccines with parents in ways that present a clear message of the importance of both vaccines.

Acknowledgements The authors thank Alexandra Gasparova, MS, Justin Tauscher, $\mathrm{PhD}$, and Melissa Watson for assistance with survey distribution and focus group moderation. We also thank the Alachua County School District and Alachua County Health Department for their continued support of infection control and prevention.

Author Contributions EJR: wrote the manuscript, analyzed data. KAR: design of the research study, data collection, and provided substantial revisions to the content of the manuscript. RML: design of the research study, data collection and provided substantial revisions to the content of the manuscript. CAH: helped with the design of the research study, and provided substantial revisions to the methods of the manuscript. AY: analyzed qualitative data, and provided substantial revisions to the methods of the manuscript. MDL: developed data analysis plan, consulted on data analysis, and substantial revisions to the content of the results. AR: data collection, and provided substantial revisions to the content of the manuscript. SASS: design of the research study, data collection, helped with manuscript writing and contributed substantial revisions to the content of all sections of the manuscript.

Funding The research reported in this publication received financial support from Pfizer, Inc. However, Pfizer was not involved with or influenced any aspect of the study/initiative/activity supported by this grant.

\section{Compliance with Ethical Standards}

Conflict of interest The authors declare that they have no known competing financial interests or personal relationships that could have appeared to influence the work reported in this paper.

Open Access This article is licensed under a Creative Commons Attribution 4.0 International License, which permits use, sharing, adaptation, distribution and reproduction in any medium or format, as long as you give appropriate credit to the original author(s) and the source, provide a link to the Creative Commons licence, and indicate if changes were made. The images or other third party material in this article are included in the article's Creative Commons licence, unless indicated otherwise in a credit line to the material. If material is not included in the article's Creative Commons licence and your intended use is not permitted by statutory regulation or exceeds the permitted use, you will need to obtain permission directly from the copyright holder. To view a copy of this licence, visit http://creativecommons.org/licenses/by/4.0/.

\section{References}

1. MacNeil, J. R., Blain, A. E., Wang, X., \& Cohn, A. C. (2017). Current epidemiology and trends in meningococcal diseaseUnited States, 1996-2015. Clinical Infectious Diseases, 66(8), 1276-1281. https://doi.org/10.1093/cid/cix993.

2. Mbaeyi, S. A., Joseph, S. J., Blain, A., Wang, X., Hariri, S., \& MacNeil, J. R. (2019). Meningococcal disease among collegeaged young adults: 2014-2016. Pediatrics, 143(1), e20182130. https://doi.org/10.1542/peds.2018-2130.

3. Mbaeyi, S. A., Blain, A., Whaley, M. J., Wang, X., Cohn, A. C., \& MacNeil, J. R. (2018). Epidemiology of meningococcal disease outbreaks in the United States, 2009-2013. Clinical Infectious Diseases, 68(4), 580-585. https://doi.org/10.1093/cid/ciy548.

4. Wang, B., Santoreneos, R., Giles, L., Afzali, H. H., \& Marshall, H. (2019). Case fatality rates of invasive meningococcal disease by serogroup and age: A systematic review and meta-analysis. Vaccine, 37(21), 2768-2782. https://doi.org/10.1016/j.vacci ne.2019.04.020.

5. Burman, C., Serra, L., Nuttens, C., Presa, J., Balmer, P., \& York, L. (2019). Meningococcal disease in adolescents and young adults: A review of the rationale for prevention through vaccination. Human Vaccines \& Immunotherapeutics, 15(2), 459-469. https://doi.org/10.1080/21645515.2018.1528831.

6. Patton, M. E., Stephens, D., Moore, K., \& MacNeil, J. R. (2017). Updated recommendations for use of MenB-FHbp serogroup B meningococcal vaccine-advisory committee on immunization practices, 2016. Morbidity and Mortality Weekly Report, 66(19), 509-513. https://doi.org/10.15585/mmwr.mm6619a6.

7. Elam-Evans, L. D., Yankey, D., Singleton, J. A., Sterrett, N., Markowitz, L. E., Williams, C. L., et al. (2020). National, regional, state, and selected local area vaccination coverage among adolescents aged 13-17 years-United States, 2019. MMWR: Morbidity and Mortality Weekly Report, 69(33), 1109-1116. https://doi. org/10.15585/mmwr.mm6933a1. 
8. McRee, A. L., Reiter, P. L., \& Brewer, N. T. (2010). Vaccinating adolescent girls against human papillomavirus-who decides? Preventive Medicine, 50(4), 213-214. https://doi.org/10.1016/j. ypmed.2010.02.001.

9. Jackson, C., Yarwood, J., Saliba, V., \& Bedford, H. (2017). UK parents' attitudes towards meningococcal group B (MenB) vaccination: A qualitative analysis. British Medical Journal Open, 7(4), e012851. https://doi.org/10.1136/bmjopen-2016-012851.

10. Mameli, C., Faccini, M., Mazzali, C., Picca, M., Colella, G., Duca, P. G., et al. (2014). Acceptability of meningococcal serogroup B vaccine among parents and health care workers in Italy: A survey. Human Vaccines \& Immunotherapeutics, 10(10), 3004-3010. https://doi.org/10.4161/21645515.2014.971602.

11. Marshall, H., Clarke, M., \& Sullivan, T. (2014). Parental and community acceptance of the benefits and risks associated with meningococcal B vaccines. Vaccine, 32(3), 338-344. https://doi. org/10.1016/j.vaccine.2013.11.042.

12. Morrone, T., Napolitano, F., Albano, L., \& Di Giuseppe, G. (2017). Meningococcal serogroup B vaccine: Knowledge and acceptability among parents in Italy. Human Vaccines \& Immunotherapeutics, 13(8), 1921-1927. https://doi. org/10.1080/21645515.2017.1313940.

13. Basta, N. E., Becker, A. B., Li, Q., \& Nederhoff, D. (2019). Parental awareness of meningococcal B vaccines and willingness to vaccinate their teens. Vaccine, 37(4), 670-676. https:// doi.org/10.1016/j.vaccine.2018.11.078.

14. Srivastava, A., Dempsey, A., Galitsky, A., Fahimi, M., \& Huang, L. (2020). Parental awareness and utilization of meningococcal serogroup B vaccines in the United States. BMC Public Health, 20(1), 1109. https://doi.org/10.1186/s12889-020-09181-8.

15. Brewer, N. T., Chapman, G. B., Gibbons, F. X., Gerrard, M., McCaul, K. D., \& Weinstein, N. D. (2007). Meta-analysis of the relationship between risk perception and health behavior: The example of vaccination. Health Psychology, 26(2), 136-145. https://doi.org/10.1037/0278-6133.26.2.136.

16. Brewer, N. T., \& Fazekas, K. I. (2007). Predictors of HPV vaccine acceptability: A theory-informed, systematic review. Preventive Medicine, 45(2), 107-114. https://doi.org/10.1016/j. ypmed.2007.05.013.

17. Dorell, C., Yankey, D., Kennedy, A., \& Stokley, S. (2013). Factors that influence parental vaccination decisions for adolescents, 13 to 17 years old: National immunization surveyteen, 2010. Clinical Pediatrics, 52(2), 162-170. https://doi. org/10.1177/0009922812468208.

18. Glanz, K., Rimer, B. K., \& Viswanath, K. (2008). Health behavior and health education: Theory, research, and practice. Hoboken: Wiley.

19. Staras, S. A. S., Vadaparampil, S. T., Patel, R. P., \& Shenkman, E. A. (2014). Parent perceptions important for HPV vaccine initiation among low income adolescent girls. Vaccine, 32(46), 6163-6169. https://doi.org/10.1016/j.vaccine.2014.08.054.

20. Iafrate, R. P., Lipori, G. P., Harle, C. A., Nelson, D. R., Barnash, T. J., Leebove, P. T., et al. (2016). Consent2Share: An integrated broad consenting process for re-contacting potential study subjects. Journal of Clinical and Translational Research, 2(4), 113-122.

21. Centers for Disease Control and Prevention. (2019). Meningococcal B Vaccine: What you need to know. Retrieved October 13, 2020 from https://www.cdc.gov/vaccines/hcp/vis/vis-state ments/mening-serogroup.pdf.

22. Centers for Disease Control and Prevention. (2019). Meningococcal Vaccination: What everyone should know. Retrieved October 13, 2020 from https://www.cdc.gov/vaccines/vpd/ mening/public/index.html.

23. National Foundation for Infectious Diseases (2019) Meningococcal disease: Focus in serogroup B. Retrieved October 13, 2020 from https://www.nfid.org/infectious-diseases/meningococcaldisease-focus-on-serogroup-b/.

24. Nvivo Qualitative Data Analysis Software [computer program]. (2018). Version 12: QSR International Pty Ltd.

25. Kuckartz, U. (2019). Qualitative text analysis: A systematic approach. In G. Kaiser \& N. Presmeg (Eds.), Compendium for early career researchers in mathematics education (pp. 181-197). Cham: Springer.

26. Harris, P. A., Taylor, R., Minor, B. L., Elliott, V., Fernandez, M., O'Neal, L., et al. (2019). The REDCap consortium: Building an international community of software platform partners. Journal of Biomedical Informatics, 95, 103208. https://doi.org/10.1016/j. jbi.2019.103208.

27. Harris, P. A., Taylor, R., Thielke, R., Payne, J., Gonzalez, N., \& Conde, J. G. (2009). Research electronic data capture (REDCap) - a metadata-driven methodology and workflow process for providing translational research informatics support. Journal of Biomedical Informatics, 42(2), 377-381. https://doi. org/10.1016/j.jbi.2008.08.010.

28. Health Resources and Services Administration. (2018). National survey of Children's Heath Questionnaire. Retrieved October 13, 2020 from https://www.childhealthdata.org/learn-about-the-nsch/ surveyinstruments.

29. MacDougall, D. M., Halperin, B. A., Langley, J. M., MacKinnonCameron, D., Li, L., \& Halperin, S. A. (2016). Knowledge, attitudes, beliefs, and behaviors of parents and healthcare providers before and after implementation of a universal rotavirus vaccination program. Vaccine, 34(5), 687-695. https://doi.org/10.1016/j. vaccine.2015.09.089.

30. National Cancer Center. (2014). Health Information National Trends Survey. Retrieved October 13, 2020 from https://hints .cancer.gov/data/survey-instruments.aspx.

31. Centers for Disease Control and Prevention. (2011). Updated recommendations for use of meningococcal conjugate vaccinesAdvisory Committee on Immunization Practices (ACIP), 2010. Morbidity and Mortality Weekly Report, 60(3), 72-76.

32. Rosenthal, S. L., Weiss, T. W., Zimet, G. D., Ma, L., Good, M. B., \& Vichnin, M. D. (2011). Predictors of HPV vaccine uptake among women aged 19-26: Importance of a physician's recommendation. Vaccine, 29(5), 890-895. https://doi.org/10.1016/j. vaccine.2009.12.063.

33. Florida Department of Health Division of Disease Control and Health Protection Bureau of Communicable Disease Immunization Section. (2019). FL SHOTS TM. Retrieved October 13, 2020 from https://www.flshotsusers.com/.

34. Kennedy, A., Stokley, S., Curtis, C. R., \& Gust, D. (2012). Limited awareness of vaccines recommended for adolescents and other results from two national consumer health surveys in the United States. Journal of Adolescent Health, 50(2), 198-200. https://doi. org/10.1016/j.jadohealth.2011.04.017.

35. Wong, C. A., Taylor, J. A., Wright, J. A., Opel, D. J., \& Katzenellenbogen, R. A. (2013). Missed opportunities for adolescent vaccination, 2006-2011. Journal of Adolescent Health, 53(4), 492-497. https://doi.org/10.1016/j.jadohealth.2013.05.009.

36. Hirth, J. M., Fuchs, E. L., Chang, M., Fernandez, M. E., \& Berenson, A. B. (2019). Variations in reason for intention not to vaccinate across time, region, and by race/ethnicity, NIS-teen (2008-2016). Vaccine, 37(4), 595-601. https://doi.org/10.1016/j. vaccine.hesitancy.

37. Roberts, J. R., Thompson, D., Rogacki, B., Hale, J. J., Jacobson, R. M., Opel, D. J., et al. (2015). Vaccine hesitancy among parents of adolescents and its association with vaccine uptake. Vaccine, 33(14), 1748-1755. https://doi.org/10.1016/j.vacci ne.2015.01.068.

38. Strelitz, B., Gritton, J., Klein, E. J., Bradford, M. C., Follmer, K., Zerr, D. M., et al. (2015). Parental vaccine hesitancy and 
acceptance of seasonal influenza vaccine in the pediatric emergency department. Vaccine, 33(15), 1802-1807. https://doi. org/10.1016/j.vaccine.2015.02.034.

39. Williams, S. E. (2014). What are the factors that contribute to parental vaccine-hesitancy and what can we do about it? Human Vaccines \& Immunotherapeutics, 10(9), 2584-2596. https://doi. org/10.4161/hv.28596.

40. Dorell, C., Yankey, D., \& Strasser, S. (2011). Parent-reported reasons for nonreceipt of recommended adolescent vaccinations, national immunization survey: Teen, 2009. Clinical Pediatrics (Phila), 50(12), 1116-1124. https://doi.org/10.1177/0009922811 415104.

41. Dorell, C. G., Jain, N., \& Yankey, D. (2011). Validity of parentreported vaccination status for adolescents aged 13-17 years: National immunization survey-teen, 2008. Public Health Reports, 126(2_suppl), 60-69. https://doi.org/10.1177/00333549111260s 208.

42. Stupiansky, N. W., Zimet, G. D., Cummings, T., Fortenberry, J. D., \& Shew, M. (2012). Accuracy of self-reported human papillomavirus vaccine receipt among adolescent girls and their mothers. Journal of Adolescent Health, 50(1), 103-105. https://doi. org/10.1016/j.jadohealth.2011.04.010.

43. Kempe, A., Allison, M. A., MacNeil, J. R., O'Leary, S. T., Crane, L. A., Beaty, B. L., et al. (2018). Knowledge and attitudes regarding category B ACIP recommendations among primary care providers for children. Academic Pediatrics, 18(7), 763-768. https:// doi.org/10.1016/j.acap.2018.04.005.
44. Kempe, A., Allison, M. A., MacNeil, J. R., O'Leary, S. T., Crane, L. A., Beaty, B. L., et al. (2018). Adoption of serogroup B meningococcal vaccine recommendations. Pediatrics, 142(3), e20180344. https://doi.org/10.1542/peds.2018-0344.

45. Gargano, L. M., Herbert, N. L., Painter, J. E., Sales, J. M., Morfaw, C., Rask, K., et al. (2013). Impact of a physician recommendation and parental immunization attitudes on receipt or intention to receive adolescent vaccines. Human Vaccines \& Immunotherapeutics, 9(12), 2627-2633. https://doi.org/10.4161/hv.25823.

46. Gilkey, M. B., Calo, W. A., Moss, J. L., Shah, P. D., Marciniak, M. W., \& Brewer, N. T. (2016). Provider communication and HPV vaccination: The impact of recommendation quality. Vaccine, 34(9), 1187-1192. https://doi.org/10.1016/j.vaccine.2016.01.023.

47. Lu, P.-J., Yankey, D., Jeyarajah, J., O'Halloran, A., Meyer, S. A., Elam-Evans, L. D., et al. (2017). Impact of provider recommendation on Tdap vaccination of adolescents aged 13-17 years. American Journal of Preventive Medicine, 53(3), 373-384. https ://doi.org/10.1016/j.amepre.2017.03.022.

Publisher's Note Springer Nature remains neutral with regard to jurisdictional claims in published maps and institutional affiliations. 